\title{
La enseñanza de la gramática como práctica reflexiva: aproximación histórica*
}

\section{Grammar teaching as a reflective practice: a historical approach}

\section{M. ' José García Folgado}

Universitat de València - GIEL

garfolma@uv.es

ORCID ID: http://orcid.org/0000-0002-5124-7471

DOI: $10.17398 / 1988-8430.35 .2 .15$

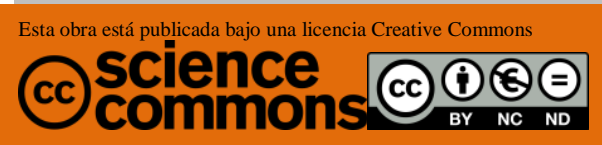

Fecha de recepción: 30/05/2021 Fecha de aceptación: 17/07/20201

García Folgado, Ma J. (2022). La enseñanza de la gramática como práctica reflexiva: aproximación histórica. Tejuelo, 35(2), 15-44.

Doi: https://doi.org/10.17398/1988-8430.35.2.15

\footnotetext{
* Este trabajo se ha realizado en el marco del Proyecto de Investigación LinPePrensa, Ideas lingüísticas y pedagógicas en la prensa española del siglo XIX (ref. PGC2018-098509-B-I00), concedido en la convocatoria de 2018 de proyectos de I+D de «Generación de conocimiento» (Ministerio de Ciencia, Innovación y Universidades).
} 
Resumen: Si bien históricamente en España se ha dado una persistente tendencia hacia las prácticas escolares basadas en la instrucción gramatical explícita, desde el siglo XIX, pedagogos, gramáticos y maestros (a través de la prensa, en las conferencias pedagógicas, en sus manuales...), entre otros, destacan los beneficios de una enseñanza orientada a promover la reflexión sobre las formas linguiísticas. La mayoría de estos autores se inscribe en una línea que acepta la gramática no como un a priori que permite el dominio correcto de la lengua sino como una herramienta de análisis y reflexión a partir del trabajo con la lengua oral y escrita. En este trabajo nos aproximamos a esa corriente, a partir del análisis de las ideas sobre la enseñanza de la lengua primera de tres autores de especial relevancia en la España de la segunda mitad del XIX y el primer tercio del XX: Mariano Carderera, José de Caso y Alexandre Galí.

Palabras clave: Gramática; reflexión metalingüística; métodos de enseñanza; historia de la enseñanza; siglo XIX.

\begin{abstract}
Although, historically, there has been a persistent tendency towards school practices based on explicit grammatical instruction in Spain, since the 19th century, pedagogues, grammarians, and teachers, among others, (through the media, in pedagogical conferences, and in their manuals, etc.) have highlighted the benefits of teaching aimed at promoting reflection on linguistic forms. Most of these authors follow a line that accepts grammar, not as an a priori matter, which allows the correct mastery of the language, but as a tool for analysis and reflection based on working with oral and written language. In this paper, we approach this trend by analysing the ideas of three authors on the teaching of first languages, who were of special relevance in Spain during the second half of the 19th century and the first third of the 20th century: Mariano Carderera, José de Caso and Alexandre Galí.
\end{abstract}

\section{Keywords:}

Grammar; metalinguistic reflection; teaching methods; history of teaching; 19th century. 


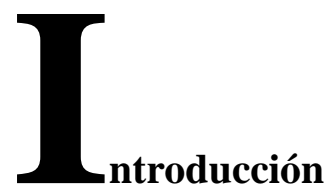

Históricamente en el ámbito hispánico, en los planes de estudio y libros de texto de la escuela primaria y secundaria, así como en las prácticas dominantes en la enseñanza de la lengua primera se ha producido de manera generalizada una identificación entre enseñar lengua y enseñar gramática, podríamos decir hasta el último cuarto del siglo XX (véase Fontich y García Folgado, 2018), si bien es una concepción que subyace en la actualidad en las prácticas de muchos docentes, como se ha puesto de manifiesto en diferentes investigaciones realizadas en España en la última década (véase, por ejemplo, Fontich y Camps, 2015; Bastons, Comajoan, Guasch y Ribas, 2017). Esto es, el código disciplinar de la materia Gramática castellana, Lengua castellana, Lengua española, Idioma nacional, etc. -entendido este, como señala Cuesta, como "una tradición social configurada históricamente y compuesta de un conjunto de ideas, valores, suposiciones y rutinas, que legitiman la función educativa atribuida a" en nuestro caso, la lengua primera "y que regulan el orden de la práctica de su enseñanza" (Cuesta 2009, p. 20)- se ha anclado en la enseñanza de la gramática explícita y su terminología técnica. No obstante, desde mediados del siglo XIX y especialmente en el primer cuarto del siglo $\mathrm{XX}$, encontramos voces que cuestionan este papel nuclear de la 
gramática. En este trabajo nos vamos a detener en algunas de esas voces discrepantes para mostrar que existe en la tradición pedagógica española una importante corriente que desplaza la gramática del centro de la enseñanza de la lengua para incidir de manera preferente en la reflexión sobre los hechos linguísticos como fuente de conocimiento a partir del trabajo con la lengua oral y escrita.

Vamos a limitarnos cronológicamente al periodo comprendido entre mediados del siglo XIX y el primer tercio del siglo $\mathrm{XX}$, que corresponde de manera amplia al nacimiento y consolidación del sistema educativo nacional en España, y nos vamos a centrar en tres autores como representantes de tres perspectivas y momentos científicopedagógicos diferentes: Mariano Carderera (1816-1892), al que situamos en la segunda mitad del XIX como representante del pensamiento pedagógico oficial español decimonónico así como de la pedagogía normalista (Vicen Ferrando, 1999); José de Caso (18501928), profesor de la Institución Libre de Enseñanza desde prácticamente sus inicios, en 1876, una institución privada creada por un grupo de catedráticos profundamente comprometidos con la renovación pedagógica española y fuertemente inspirados por el Krausismo y, por último, Alexandre Galí (1886-1969), quien desarrolla su obra en el primer tercio del siglo $\mathrm{XX}$, vinculada al ideario pedagógico de la Escuela Nueva.

\section{1.- La reflexión sobre el uso en la enseñanza de la lengua 1.1.- El contexto epistemológico}

Como ya se ha indicado, si bien es cierto que en las prácticas presentes en la escuela se ha mantenido la gramática como una de las piedras angulares del estudio de la lengua, su aceptación no ha sido unánime $\mathrm{y}$, aun siendo reconocido su valor para el desarrollo correcto de las habilidades linguísticas, se critica abiertamente el método de enseñanza. Dice José de Caso al respecto

El estudio del idioma nacional figura, sin disputa, en el número de los que más capitales reformas exigen entre nosotros. Relegado á un lugar secundario, mirado como ocupación propia de niños exclusivamente, 
y no muy atendido ni aun en esta edad de la vida, redúcese al aprendizaje de unas cuantas definiciones, no siempre exactas, y de un cúmulo de reglas, artificiales y arbitrarias muchas veces, pasivamente recibidas y mecánicamente repetidas, en las cuales ninguna enseñanza viva y fecunda se encierra sobre la lengua que se aprende, nada que pueda servir de guía al que la estudia para entrar gradualmente en lo sucesivo en posesión del idioma y poder servirse de él con algún arte y libertad (Caso, 1879, p. 210).

En esa misma línea, son bien conocidas las críticas de autores como Miguel de Unamuno (1907), Rodolfo Lenz (1912) o Américo Castro (1921), entre otros (Bargalló, 2007); no obstante, no es tan conocido que existe una larga tradición de gramáticos, pedagogos, maestros, etc. que postulan la necesaria vinculación entre el uso de la lengua y su aprendizaje y aceptan la gramática no como un a priori que permite el correcto dominio linguístico sino como una herramienta de análisis y reflexión. Esto es, se aboga por un camino didáctico que vaya de la lengua a la gramática.

Un autor fundamental en este planteamiento es el pedagogo suizo Johann Heinrich Pestalozzi (1746-1827), quien desarrolla sus ideas sobre la enseñanza de la lengua en dos obras fundamentalmente: en la carta $7^{\mathrm{a}}$ de su libro Cómo Gertrudis enseña a sus hijos (1801) y en el Libro de las madres (1803). Pestalozzi plantea un método de tipo inductivo, el denominado método intuitivo, que consiste en "someter las formas de instrucción a las leyes eternas por las cuales se eleva el espíritu humano de las intuiciones sensibles á los conceptos exactos" (Luzuriaga, 1925, p. 8). Este método se inspira en los planteamientos empiristas de Locke y en las ideas de Rousseau y coloca en el centro del proceso educativo al niño, sus intereses y descubrimientos. Desde esa idea, el método consiste en llevar al alumnado de lo conocido a lo desconocido, de los hechos a sus leyes y causas, de lo concreto y particular a lo abstracto y general, a través de la observación, de la comparación y del análisis de la realidad.

El pensamiento pedagógico de Pestalozzi tuvo una gran influencia en España durante todo el siglo XIX y el primer tercio del XX (Sureda, 1985; Viñao, 1997) y la metodología intuitiva fue clave en 
la enseñanza primaria, en todas las materias de estudio (de hecho, fue el tema central en el primer Congreso Pedagógico Nacional celebrado en Madrid, en 1882). Especialmente importante para la difusión de sus ideas fue la formación de los maestros y maestras, tanto a partir de la inclusión de sus planteamientos en los libros de texto para las Escuelas Normales como el Curso elemental de Pedagogía de Avendaño y Carderera (1850) -aprobado como libro oficial en 1852, 1856, 1861, 1864, 1867 y recomendado de nuevo durante el primer decenio de la Restauración (Rabazas, 2008, p. 287)-, como las traducciones de las obras de sus seguidores para las Escuelas Normales, como la de M. A. Jullien (1862).

Los autores analizados se sitúan en ese marco y expresan la idea de que el aprendizaje de la lengua ha de ser principalmente práctico (intuitivo, reflexivo) y colocan en un segundo lugar las cuestiones teóricas, como se puede apreciar en las tres citas que siguen:

Por la intuicion y la reflexión [...] se aprende la lengua materna hasta comprenderla y hacer uso de ella y hablar correctamente. Mas para una buena educacion no basta proceder con exactitud, sino que es indispensable darse cuenta de las operaciones y conocer las reglas á que está sujeta la manera de proceder (Carderera, 1865, p. 264)

La enseñanza del idioma debe tener por base la práctica, debe fundarse en un sistema de ejercicios que adiestren al que aprende en el uso de la lengua. ¿Y la teoría? La teoría nace de la reflexión sobre ese uso, y en él tiene su destino inmediato, puesto que a dirigirlo se consagra. Surgirá, pues, al paso con la práctica misma, y llegará en cada momento hasta donde lo consienta la experiencia anterior del alumno en el conocimiento y manejo del idioma (Caso, 1889, p. 15).

El millor procediment per a trencar la formalitat de la Gramàtica és enfrontar a l'infant amb els fets de llengua tal com ens són donats en la llengua viva: la llengua de la parla usual i la llengua literària. Res de classificacions ni definicions a priori. Captar el fet d'expressió en la seua forma viva i anar-lo seguint a poc a poc per veure què ens dóna l'anàlisi o estudi de cada contingut expressiu (Galí, 1935, p. 8).

Pese a la similitud del planteamiento, nos interesa destacar que cada uno de estos autores parte de bases teóricas diferentes acerca del 
hecho lingüístico y el aprendizaje. Carderera se sitúa, desde el punto de vista de la lingüística, en la corriente que vincula la lengua con el pensamiento, de raigambre lógica y que entronca con las ideas de los gramáticos filósofos como Condillac, Destutt o Laromiguière, entre otros. Desde esa perspectiva, el análisis de la expresión es concebido como el paso previo y necesario para el análisis del pensamiento (véase Calero Vaquera, 2008 y 2015):

El estudio de la lengua es el fundamento de la cultura intelectual, y el que no aprende el arte de la palabra no puede aprender el arte del pensamiento, que es lo que distingue esencialmente al hombre de los demás seres [...] La lengua es el instrumento universal de que se vale el espíritu, no solo para manifestar sus pensamientos, sino para fijarlos y circunscribirlos por sí mismo; porque la lengua representa á la vez, por medio de las palabras, el dominio completo de las ideas (Carderera, 1855, p. 412).

Desde el punto de vista didáctico, sus ideas sobre la enseñanza de la lengua proceden tanto de Pestalozzi como, principalmente, de Gregoire Girard (1765-1850), ambos ampliamente citados en sus obras pedagógicas. Si más arriba hemos hablado de la importancia de Pestalozzi en el pensamiento pedagógico español, Girard, quien adapta el método del pedagogo suizo dando preferencia a los aspectos lingüísticos (Vilanou y García Farrero, 2016), es el principal autor de referencia para la metodología de enseñanza de la lengua en España ${ }^{1}$. Aunque su obra, De l'enseignement régulier de la langue maternelle (1844), no se traduce hasta 1876, de la mano de Prudencio Solís y Miguel, profesor de la Escuela Normal de Valencia, sus ideas eran ya muy conocidas en ese momento, tanto por la circulación de sus obras en lengua original como, muy especialmente, a través de la formación de maestros. Asimismo, sus planteamientos aparecen de manera reiterada

\footnotetext{
${ }^{1}$ El peso tanto de Pestalozzi como de Girard en la didáctica de la lengua en la segunda mitad del siglo XIX se evidencia en el hecho de que en los exámenes de reválida de los títulos del magisterio de primera enseñanza se incluía en esa época un tema relativo a la metodología específica del estudio de la lengua según sus teorías (véase, por ejemplo, el Reglamento y cuestionarios oficiales para los exámenes de reválida de los títulos de Magisterio de Primera Enseñanza, Madrid: Imprenta del Colegio Nacional de Sordo-mudos y ciegos, 1885).
} 
en la prensa del Magisterio y se discuten en las conferencias profesionales $^{2}$.

En la propuesta de Girard, recogida por Carderera, se fomenta principalmente el estudio del vocabulario, de la sintaxis y de la composición, a través de ejercicios orales y escritos, si bien el objetivo principal es la lengua escrita. La finalidad última dada a la formación lingüística es servir al desarrollo de todas las facultades intelectuales y al perfeccionamiento moral del individuo. Esto se puede observar en las figuras 1 y 2 que aparecen a continuación, en las que se explicitan los contenidos que se deben trabajar por cursos o grados:

\section{Figura 1}

Cuadro sinóptico del curso de lengua materna. $1^{\circ}$ grado

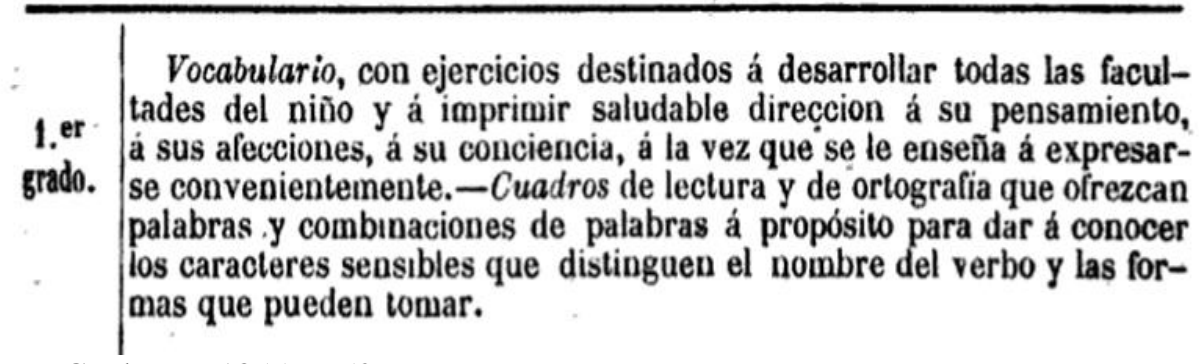

Fuente: Carderera, 1855, p. 526

\footnotetext{
${ }^{2}$ Véase, por ejemplo, las sesiones del 26 de febrero y el 11 de marzo de 1888 de la Conferencia de Maestros de Valencia, destinadas a la enseñanza de la legua en las escuelas primarias, publicadas entre los meses de abril y mayo en periódicos como $\mathrm{La}$ Unión del Magisterio, n. ${ }^{\circ} 13$ a 16; el Boletín de primera enseñanza, n. $^{\circ} 13$ a 15, o El Magisterio Balear, n. ${ }^{\circ}$ 13-15, entre otros).
} 


\section{Figura 2}

Cuadro sinóptico del curso de lengua materna. $3^{\circ}$ grado

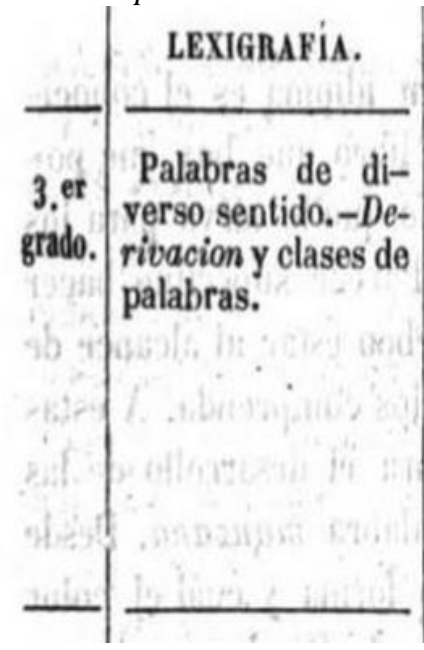

sINTÁxis.

Frase de dos miembros formada por un objeto, un explicativo, un determinativo, por adicion, por oposicion, por alternativa, por comparacion. - Frase causal final, condicional. - Conjugacion del condicional y del subjuntivo.

Fuente: Carderera, 1855 , p. 527

COMPOSICION.

Narraciones.-Cartas familiares, dando el maestro el asunto y las ideas principales.

Caso, por su parte, se sitúa ya en una perspectiva epistemológica distinta, alejada de los planteamientos de la gramática general y la lógica, y enmarcada en la lingüística de finales del XIX, determinada por el comparatismo y por el nacimiento de la semántica moderna (aunque no rechaza del todo los planteamientos anteriores). Asimismo, este autor está en la línea de lingüistas como Schuchardt y, muy especialmente, Bréal, quienes conciben la lengua como un hecho social $^{3}$ :

Verdad es que el lenguaje no obedece de una manera rígida, inflexible y uniforme á las leyes del pensamiento, sino que, reflejo fiel de toda nuestra vida, recibe dócilmente la impresión de las múltiples influencias que intervienen en su desarrollo y se despliega en una rica

\footnotetext{
3 Tanto Schuchardt como Bréal, entre muchos otros lingüistas finiseculares, asumen, tal y como indica Sornicola, "the Humboldtian idea whereby the «speaking subject» and his linguistic activity, understood as $\delta v$ vauls, energeia, are the ultimate foundation both of the reality of languages and of their scholarly study", en un movimiento de reacción contra "the «exterior observation of the forms of language» and on the reconstruction of «laws» governing change in sounds, words, inflections and syntax understood as material elements, having no relationship with the individuals who use them (Bréal, [1866] 1877: 248-249)" (2014, pp. 30-31).
} 
variedad de formas correspondientes á las diversas esferas de la individualidad humana; pero que no obedezca á dichas leyes de una manera inflexible y uniforme, no quiere decir que se sustraiga á las mismas: significa tan sólo que en cada idioma se cumplen de un modo original (Caso, 1879, p. 321).

Su pensamiento didáctico sobre la lengua está firmemente enraizado en las ideas de Michel Bréal (1842-1915), autor de gran repercusión en la España de fin de siglo, tal y como da cuenta el gran eco que sus conferencias tienen en la prensa profesional del magisterio ${ }^{4}$. Una de las ideas principales de este autor es que es inútil aprender una lengua viva como se aprenden las muertas, porque su objeto esencial es la comunicación: "on ne possède une langue" dice Bréal "que quand on peut la parler. Il faut pouvoir à tout instant la tirer de son esprit et la produire au dehors: l'intelligence d'un texte ne suffit pas. Le maniement d'une langue est une forme de l'activité [...]" (Ministère du Commerce, de l'Industrie et des Colonies, 1889, p. 25). De ahí que la realidad y sus exigencias prácticas se constituyan como el motor del aprendizaje y la gramática ocupe el último lugar; la frase "se debe llegar á la gramática por la lengua y no a la lengua por la gramática”, que Bréal incluye en una conferencia pronunciada en 1876 titulada "La enseñanza de la lengua francesa" (véase Boutan, 1998, cap. 5), se convierte, de hecho, casi en un aforismo, largamente repetido en los escritos pedagógicos sobre la lengua en España.

Desde esa perspectiva, forman parte del programa que presenta Caso para la educación linguiística en la Institución Libre de Enseñanza, principalmente, la conversación cotidiana, las narraciones y el trabajo mental con los niños para que se den cuenta de qué piensan y cómo lo expresan; esto es, la prioridad se da a la lengua oral, aunque no se deja

\footnotetext{
${ }^{4}$ Así, por ejemplo, se traduce en diferentes cabeceras la conferencia impartida en 1886 en la Association Scientifique titulada "Cómo se aprenden las lenguas extranjeras" (Boletín de la Institución Libre de enseñanza, 30.4.1886 y 15.5.1886), se publican las discusiones en torno al tema de la enseñanza lingüística que se produjeron en el Congreso Internacional de la Enseñanza Superior y de la Enseñanza Secundaria, celebrado en París, coincidiendo con la Exposición Universal en 1889 (por ejemplo, en el Boletín de Primera Enseñanza, 29.10.1889 o Boletín de la Institución Libre de Enseñanza, 30.6.1890, entre otros), etc.
} 
de lado la escrita, ni mucho menos. Además, Caso, siguiendo a Bréal, otorga una gran importancia al vocabulario y, de hecho, plantea los beneficios que la comparación entre lenguas y la etimología pueden tener para su enseñanza, en un último estadio educativo (Caso, 1879, n. ${ }^{\circ}$ 68 y 69$)$.

Por último, las propuestas de Galí entroncan con la lingüística moderna de raigambre saussuriana, especialmente con la obra de Bally, Le langage et la vie de 1926, en la que se entiende que el lenguaje es vida y acción y por tanto los fenómenos linguiísticos han de ser analizados en su realidad:

Au total, nous voyons un peu mieux, sinon ce que c'est qu'une langue, $\mathrm{du}$ moins que ce qu'elle n'est pas: le langage naturelle, celui que nous parlons tous, n'est au service ni de la raison pure, ni de l'art; il ne vise ni un idéal logique, ni un idéal littéraire; sa fonction primordiale el constante n'est pas construire des syllogismes, d'arrondir des périodes, de se plier aux lois de l'alexandrin. Il est simplement au service de la vie [...] (Bally, 1926, p. 14).

En esa línea, la lengua, para Galí, es un hecho viviente que se adapta a las condiciones de la vida:

[...] la llengua separada de l'acte viu que la produeix no és res. L'individu aplica, utilitza uns elements, un material que li són donàs, però ell, amb el foc del seu esperit, els dóna una virtut nova, una vida nova, como si creés una llengua nova (1931a, p. 17).

Asimismo, plantea una gradación en esos fenómenos que parte de las propuestas de Sechehaye, en su Essai sur la structure logique de la phrase (1929). Desde el punto de vista pedagógico, como ya se ha indicado, Galí se vincula con el movimiento de la Escuela Nueva (Pozo Andrés, 2003-2004) que, según su propio pensamiento, planteaba que la enseñanza se tenía que ajustar a la realidad viva del niño para posibilitar su incorporación a la realidad igualmente viva del mundo de los adultos (véase González Agapito, 1984, pp. 33-40).

Cabe señalar que el autor catalán se sitúa en un momento muy interesante de la historia de la enseñanza de la gramática, porque en 
esas primeras décadas del siglo $\mathrm{XX}$ se produce una eclosión de escritos que se plantean tanto el qué enseñar como el porqué y el cómo; por ejemplo, en 1916 se publica la traducción de la obra de Laura Brackenbury, La enseñanza de la gramática, realizada precisamente por una mujer de la Institución Libre de Enseñanza, Alicia Pestana; hacia los años 20, se reeditan en la colección "Ciencia y Educación" de las ediciones La Lectura los textos de Pestalozzi o el tratado sobre Girard de Gabriel Compayré, entre otros, y las revistas pedagógicas españolas en ese periodo dedican infinidad de páginas a esta cuestión.

Como hemos podido apreciar, Carderera, Caso y Galí, a grandes rasgos, son representantes de la recepción de las ideas lingüísticas y pedagógicas europeas en España a lo largo de la segunda mitad del siglo XIX y principios del XX: por una parte, se va de la gramática filosófica a la linguística moderna, con las diferencias que esas aproximaciones comportan, pero por otra, como vamos a ver en los apartados que siguen, los postulados pedagógicos que rigen sus propuestas, pese a provenir de fuentes diversas, se orientan de manera similar.

\section{2.- La lengua, la gramática y la enseñanza}

Los tres autores articulan el método de enseñanza a partir de tres premisas fundamentales, que ya están en Pestalozzi:

1) el proceso de enseñanza ha de seguir necesariamente la marcha natural del desarrollo intelectual de los niños;

2) la teoría se subordina a la práctica y

3) una cosa es la lengua y otra, su gramática.

Como ya se ha indicado, el método intuitivo y las metodologías activas en general, colocan en el centro de la acción didáctica al niño y se considera básico graduar la enseñanza en función de sus características e intereses. Esta premisa está en los tres autores, pero de manera mucho más clara en Galí, ya que es un gran conocedor de los estudios psicológicos de la época (Camps, 1997, p. 211); así, indica: "Per posseir tots els aspectes de l'ensenyament del llenguatge a l'infant, falta esbrinar de quina manera es comporta l'infant; com s'integra en la 
funció col-lectiva de la llengua que correspon al nucli social on li pertoca viure [...]" (1931a, p. 25). Esta "marcha natural" del niño implica que se organiza y gradúa la introducción de las estructuras de la lengua y las nociones gramaticales en función de los estadios del desarrollo linguiístico infantil; tal y como indica Galí: "En realitat, amb els exercicis, no fem sinó repetir pas a pas i d'una manera explícita els mateixos exercicis que l'infant realitza implícitament quan aprèn a parlar" (1935a, p. 10).

En segundo lugar, la subordinación de la teoría a la práctica es fundamental en este planteamiento, ya que se entiende la lengua como un saber práctico y se ve como poco útil partir de los conceptos teóricos para llegar al dominio práctico del idioma; así se aprecia en las palabras de Caso:

La enseñanza de la lengua tiene un doble carácter: ha de ser teórica y práctica á la par; y lo segundo más que lo primero, si se admite que aun los principios y leyes, que han de servir de guía en el análisis práctico del idioma, un niño no puede llegar á conocerlos sino mediante la práctica misma, merced á ese mismo análisis, es decir, empezando por descubrirlos en algunos de los casos á que se aplican, para aplicarlos él después á todos los semejantes, pero nunca como fruto de una exposición puramente teórica y abstracta (Caso, 1879, p. 98).

En cuanto al último aspecto, aunque los tres están entrelazados, es fundamental la idea de que hay que diferenciar la lengua de la gramática. Esto es, no se pretende la eliminación de la gramática como contenido, sino que se plantea una gradación en el aprendizaje que vaya desde la propia lengua del niño, sometida a un proceso de observación desde una perspectiva práctica y manipulativa hasta la sistematización de lo observado por medio de la gramática. Hay que entender que, para estos autores, así como para muchos docentes del periodo, la gramática es una disciplina formal, externa al alumno y poco adecuada para los primeros años, tal y como explica Caso:

No se trata, como se ve, de torturar el idioma para convertirlo en una especie de álgebra del pensamiento, sino de tomarlo tal y como naturalmente brota bajo las exigencias de este ultimo, y promover su 
cultivo y desarrollo en esa armonía natural que con el mismo mantiene, á fin de que sea instrumento docil y flexible puesto al servicio de tales exigencias, no remora y traba á su satisfacción (1879, p. 93).

También Galí considera la gramática "una mena d'àlgebra de la qual l'infant pot aprendre més o menys les regles, les fórmules i, àdhuc, alguna demostració; però ell sempre es pregunta: ¿A què ve tot això?” (1935a, p. 5).

Se puede apreciar por las palabras de Caso y de Galí que un primer problema es la gramática en sí, el texto gramatical (véase Fontich y García Folgado, 2018). Esa gramática, en palabras de Galí es "formal, sistematitzada, estructurada en un món d'abstraccions que estan molt be, però que no tenen [...] gaires punts de contacte amb el món de l'infant" (1931a, p. 7). Carderera, que sigue a Girard en esto, resuelve la ruptura entre esa gramática formal y la gramática que demanda la enseñanza en el marco planteado introduciendo una interesante división entre lo que denomina gramática dogmática y gramática pedagógica. Así, dice:

En las dogmáticas se enseñan como verdades admitidas por los sábios las propiedades lógicas y gramaticales de las partes del discurso, y las reglas de su construcción, ya se expongan estas propiedades y estas reglas de una manera sintética, como en las antiguas gramáticas, ya de manera analítica, como en las mejores de las modernas.

Las pedagógicas, por su parte, son las que conducen al niño como por la mano a examinar su propia lengua, á reconocer el uso y el valor de las diversas partes que la componen, á apreciar por lo menos la naturaleza lógica de las ideas, y a distinguir bien los caracteres gramaticales de la palabra, según su diversa naturaleza, excitándole despues y ayudándole en el ejercicio activo de la inteligencia y habituándole al análisis, madre del verdadero saber (Carderera, 1865, p. 261).

Galí no habla explícitamente de gramática pedagógica, aunque eso subyace en su planteamiento, cuando habla de "la nova Gramàtica que ha de donar forma i ha d'ésser el centre d'orientació de totes les activitats en ordre a l'ensenyament del llenguatge" (1931a, p. 31); para él, se trata de convertirla en una disciplina adaptada a la realidad 
lingüística y a la realidad del alumnado, que permita organizar y unificar las observaciones que se realicen tras "exterioritzar el llenguatge i posar-lo com objecte de consideració i estudi” (1931a, p. $10)$.

Veamos, a continuación, como se sistematizan estas ideas en propuestas prácticas de enseñanza.

\section{2.- De la teoría a la práctica}

Como se aprecia en las citas anteriores, el camino para romper la formalidad de la gramática es el uso de la lengua y la reflexión sobre el uso. El método, que parte de la intuición en los términos señalados ya por Pestalozzi y sus seguidores, implica un procedimiento de tipo inductivo que se articula en dos fases: se inicia con los denominados ejercicios de lenguaje -aunque para Galí, este tipo de tareas deberían realizarse en todas las áreas de enseñanza, durante toda la escolarización, no solo algo restringido a los primeros años- y en un segundo momento se introduce la teoría gramatical.

\section{1.- Los ejercicios de lenguaje}

El aprendizaje inicial de la lengua oral y escrita se articula en torno a ejercicios de lenguaje, destinados a afianzar los conocimientos sobre la lengua a través de la reflexión. Carderera, explica el planteamiento a los futuros maestros en los siguientes términos:

Al niño debe enseñársele la lengua sacándole del estrecho circulo de las reglas gramaticales, para introducirle en el dominio real del pensamiento. Basta para esto estudiar la marcha que sigue la madre inspirada por la naturaleza é imitarla metodizando y dando mas extension á los ejercicios prácticos de que instintivamente se vale para que su hijo aprenda á hablar y á comprender lo que dicen los demás. En lugar de una exposicion pedantesca y de definiciones incomprensibles, se le hace observar los objetos y los nombra y los compara adquiriendo así un caudal de voces á la vez que ejercita y desarrolla las facultades de su alma. Mas adelante se le presenta una frase para hacerle distinguir las palabras de que consta y á fuerza de ejemplos y de lecciones que le interesan y agradan, descubre por si 
mismo el valor, el uso y la forma de las voces y comprende la definición (Carderera, 1865, p. 249).

Para Galí, con estos ejercicios se pretende en primer lugar, "afinar la sensibilitat per a les coses de llenguatge", para, en segundo lugar "afinar la intuïción dels fenòmens de la llengua per a penetrar-ne l'estructura i les lleis de funcionament i preparar així la introducció a la Gramàtica" (1935a, p. 4).

Se trata, sobre todo, de ejercitar el uso de la lengua, lo que debe contribuir a la mejora de la expresión, pero también, a procurar que el niño sea consciente de qué es correcto y qué no; para Carderera (1865, p. 253) el estudio práctico "hace sentir los matices más delicados de la lengua y enseña a distinguirlos sin necesidad de reglas" ya que, indica, "hablamos gracias a una especie de tacto, de sentimiento íntimo del genio de la lengua" que es resultado del uso. Por su parte, para Galí se trata de "procurar que l'infant senti el gust del llenguatge i que per pura impresió estètica sàpiga endivinar el que està bé o malament" (1931a, p. $4)^{5}$.

La figura 3 muestra un ejercicio, que sigue los planteamientos de Pestalozzi, destinado a que el alumnado no solo observe el funcionamiento de las estructuras gramaticales y abstraiga las regularidades y principios que lo rigen, sino también a que exprese sus ideas y pensamientos; como señala Carderera: "aunque los ejercicios sean prácticos no por eso deben ser mecánicos y rutinarios, sino que por medio de preguntas deben encaminarse al ejercicio de la inteligencia, al desarrollo del sentimiento y a la formacion del gusto" (1865, p. 250).

\footnotetext{
El concepto de corrección en Galí es mucho más complejo y presenta interesantísimas derivaciones ligadas a su concepción de lo que es la lengua que, entendida como ente vivo, comporta intrínsecamente la idea de variación y la crítica al modelo de lengua única, fija, etc. (véase, por ejemplo, 1931a, pp. 19-23), siguiendo, entre otros, a Vossler, cuya obra había sido divulgada en catalán por Manuel de Montolíu (Portolés, 1986).
} 


\section{Figura 3}

Ejercicios para el desarrollo del lenguaje. $6^{\circ}$ grado

Primer ejercicio. Relaciones expresadas por el verbo tener, consideradas relativamente al nombre.

Se determina con referencia al nombre las relaciones que conciernen à los objetos. Ejemplos: el ave tiene un pico, dos piés, etc.; el perro tiene un rabo, cuatro patas, elc. Con respecto al ser, puede ponerse la condicion siguiente : los son cinco, y en este caso, el niño dirá, por ejemplo, los dedos de la mano son cinco; los del pié, etc.

Segundo ejercicio. Relaciones del tener, consideradas relativainente á las cualidades de los objetos.

Dirigese á determinar los objetos por sus cualidades dominantes, ó por sus rasgos característicos.

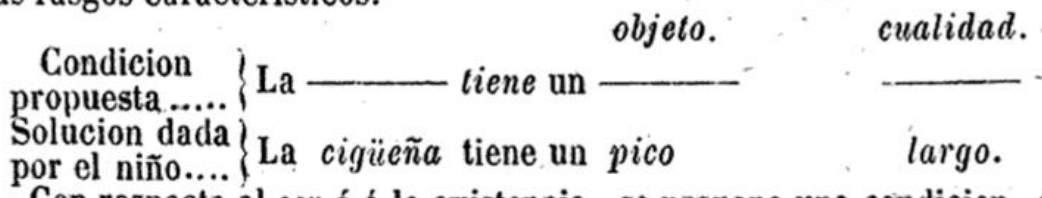

Con respecto al ser ó á la existencia, se propone una condicion, que debe cumplirse de este modo:

$$
\mathrm{El}-\overline{\text { lobo es }} \text { eruel. }
$$

Fuente: Jullien, 1862, p. 170

Las figuras 4 y 5 son fragmentos del manual de Galí, Lliçons de llenguatge ( $1^{\circ}$ grado, 7-9 años, 1931b) y en ambos casos se parte de la producción o bien de la manipulación para suscitar la reflexión. 


\section{Figura 4}

Ejercicio sobre comparaciones. Primer grado

\section{TEMA 1 (Comparacions)}

Exercici. - En aquest dibuix hi ha un carrer, un arbre i unes quantes persones. Digueu com és

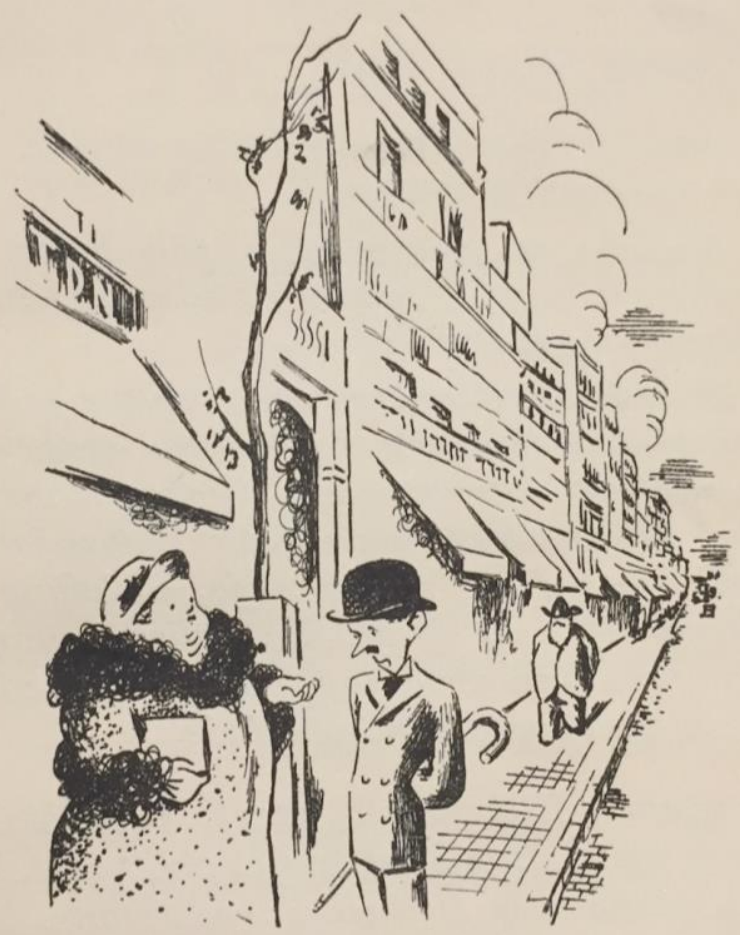

cada cosa i cada persona d'aquestes fent servir el com o el més... que. Posareu: Un carrer més llarg que... Un arbre sec com..., etc., etc.

Fuente: Galí, 1931b, p. 7 


\section{Figura 5}

Ejercicio sobre construcción de frases. Primer grado

\section{TEMA 37 (Construcció de frases)}

Llegiu aquesta frase: He vist una processó pel carrer de casa. ¿De quantes maneres correctes es pot dir?: Pel carrer de casa he vist una processó, He vist pel carrer de casa una processó.

Exercicr. - Canvieu la construcció de les frases següents, cercant per a cada una, totes les formes possibles que estiguin bé:

Nosaltres hem fet un present de fruites $i$ flors a la nostra mare amb motiu del seu sant. Aquestes nenes fan un ram molt bonic amb les roses del nostre jardí. Els mariners s'enfilaven pels pals del vaixell amb molta agilitat. Les roques brillaven totes daurades a la claror del sol ponent. Pel carrer passaven moltes colles amb les banderes desplegades. El vent bufava per les teulades de les cases amb tota la fúria.

Fuente: Galí, 1931b, pp. 50-51

Como se puede observar, los ejercicios de lenguaje ponen en primer plano el desarrollo de las habilidades cognitivas y se desplaza la capacidad analítico-taxonómica: lo importante no es que el alumno asimile la teoría y reconozca las categorías del discurso gramatical, sino que, a partir de la manipulación, se suscite la observación de los fenómenos lingüísticos; de este modo el alumno se convierte en un elemento activo en el proceso de enseñanza.

Los ejercicios de lenguaje se plantean, pues, como una iniciación a la reflexión lingüística, principalmente, desde la oralidad, orientada a que "el niño extienda su uso, en la medida más amplia posible, fuera de los límites en que se encierre al comenzar" (Caso, 1889, p. 11). 


\section{2.- La introducción de la gramática en el aula}

De estos ejercicios de lenguaje se pasa a la gramática, que se introduce con objeto de sistematizar lo aprendido. Como indica Carderera, "La práctica del lenguaje dá un conocimiento empírico de la lengua; por consiguiente, para apreciar lo aprendido y aplicado en los casos que no se hayan explicado, es indispensable hacer un estudio mas formal" (1865, p. 256) y Galí indica que esas prácticas lingüísticas iniciales "perdran la seva eficàcia si no tenen un punt d'unificació per mitjà d'una disciplina superadora $\mathrm{i}$ sistematitzadora que hauria d'ésser la Gramàtica" (1931a, p. 10; cfr. Camps 1986, p. 49).

Esa enseñanza, de nuevo, se plantea en el marco del método intuitivo y se persigue fomentar la reflexión a través del diálogo sobre los hechos lingüísticos. En la figura 6 , se presenta un modelo de conversación gramatical orientado al conocimiento y clasificación de las palabras en el que el maestro guía el trabajo de reflexión de los discípulos a través de preguntas y solo al final se introducen las nociones gramaticales (véase Carderera, 1865, pp. 266-268 para una explicación completa sobre este tipo de interacción en el aula). 


\section{Figura 6}

Ejemplo de enseñanza gramatical en las escuelas. Grado mínimo

\section{GRADO MÍNIMO. \\ Un ejercicio sobre el nombre.}

Nómbrame una cosa de la Escuela.

- Tintero (I).

Otra.

-Puntero.

Otra.

-Papel.

¿Para qué sirve el tintero?

- Para conservar la tinta.

¿Y el puntero?

- Para señalar.

¿Y el papel sirve para una cosa ó para muchas?

- Para muchas.

Dime alguna.

- Para escribir.

Nómbrame algunas cosas de la Escuela que sean de papel.

- Los libros, los carteles, los mapas.

Con la palabra tintero ise nombrará una cosa?

- Si, señor.

¿Luego la palabra tintero será un nombre?

- Si, señor.

¿Y puntero?

-Otro nombre.

¿Y papel?

-Otro nombre.

Dime nombres de cosas de la Escucla.

-Banco, mesa, pizarra.

¿Con que hemos visto que los nombres sirven para....?

- Para nombrar las cosas.

¿Tienes algún hermano?

-No, señor.

¿Y tú?

- Si, señor.

¿Cómo se llama?

-Manuel.

Y si fuera hermana ¡cómo se llamaría?
Con las palabras Manuel y Manuela ¿qué nombramos?

-Personas.

¿Será nombre la palabra Manuel?

-Sí, señor,

¿Y Manuela?

-También.

¿Luego el nombre servirá también para nombrar personas?

-.Sí, señor.

Antes decíais que el nombre servía para nombrar cosas, y ahora decís que sirve también para nombrar personas. ¿Con que para qué sirve e! nombre?

- Para nombrar cosas y personas.

Según esto, las palabras que nombran cosas y personas jcómo se llamarán?

-Nombres.

Dime nombres de algunas personas que conozcas.

-Antonio, Juan, etc.

Nombres de cosas de la cocina.

-Pucheros, tazas, etc.

De cosas de la mesa.

- Cuchara, tenedor, etc.

De cosas que se comen.

- Pan, carne, huevos, etc.

De cosas que se beben.

-Agua, vino leche, etc.

Como secomprenderá, esto se prolonga cuanto se quiera.

Fuente: Comisión de Maestros de Valencia, 1888, p. 4

De forma similar, Galí en su manual Introducció a la Gramàtica (vol. I, 1935b) plantea tres lecciones iniciales, en su caso, sobre la frase: se parte de un diálogo breve y se indica al alumnado: "cada vegada que 
Josep o Pere parlan, fan una frase. ¿Quantes frases fa cada interlocutor?" (p. 8). Después, aparece otro diálogo más complicado porque cada interlocutor no usa una única frase en cada turno y se hace observar a los niños cómo cada intervención se puede "romper" en diferentes frases. A continuación, se trabaja con fragmentos narrativos y se realizan diferentes ejercicios y solo al final de la tercera lección, se indica "la frase, doncs, és una unitat expressiva que no es pot trencar" (p. 11).

Este trabajo sobre el uso de la lengua y la gramática se plantea desde una perspectiva global, esto es, se parte de la frase para, a partir de su análisis ver cómo se integra y cómo va haciéndose compleja. Carderera plantea que

El que sabe una lengua no pronuncia voces aisladas, sino palabras, frases, juicios, pensamientos. Conoce el todo y siguiendo el principio de pasar de lo conocido á lo desconocido, de la frase debe pasar á las partes de que se compone. Esta es la marcha que debe seguirse en toda la enseñanza. [...] Conocidas las partes por medio del procedimiento analítico, se combinan los ejercicios de análisis y síntesis para descubrir y aplicar las reglas en condiciones determinadas. Estudiada por este medio una forma cualquiera del lenguaje, se presentan y se hace presentar ejemplos en que entre esta forma segun la regla ó las reglas que se hayan aprendido (1865, p. 265).

Como se puede observar en la última parte de la cita, el método que propone va del análisis a la síntesis, algo que es muy habitual en la época y aparece en muchas gramáticas escolares del periodo -las mismas, precisamente, que Carderera incluye bajo la etiqueta de "gramáticas pedagógicas" o "modernas"- que, siguiendo las pautas marcadas por la gramática filosófica, parten del todo -el pensamiento, el juicio- para llegar a sus partes y, desde ahí descubrir las ideas que están contenidas en él. La generalización de este planteamiento en la gramática escolar supone la reorganización de los contenidos: se parte de la oración y no de la palabra, con un consecuente aumento del peso de la sintaxis y la introducción de los denominados "ejercicios de análisis" (lógico y gramatical) en los manuales escolares (Calero, 2008 y 2009; Montoro del Arco y García Folgado, 2009; García Folgado, 2012). 
Caso y Galí, como se desprende de los ejemplos, lo plantean en esa misma línea analítica. El primero de ellos indica:

Familiarizando al niño desde temprano con la frase y el período, y con las operaciones de la inteligencia á que corresponden, se logrará que analice las palabras en vista del conjunto de que deben formar parte, y donde tienen su puesto y cumplida explicación; que comprenda el lugar que pertenece y el papel que toca á cada una en la oración y en el discurso; que tenga bien dispuestos y elaborados en su pensamiento dichos elementos ó materiales para la construccion á que se destina; y que entre, pues, en el examen de ésta con los datos indispensables é iniciado en aquellos principios que deben servirle de guía para darse cuenta exacta del complejo mecanismo de la expresión (1889, p. XX).

\section{De manera similar, Galí señala:}

L'infant sent la frase com un tot, aprèn de encontrar-hi en ella els elements amb les relacions que els donen la unitat essencial, i després aplica aquests elements per a dir el que es propone, d'acord a unes lleis estructurales que se li han encomanant (1935a, p. 10).

Esto, para Galí, implica una gradación en ese proceso de descubrimiento de la formación de la frase que debe seguir "pas a pas la manera com la frase s'integra i es va fent complexa" (1935a, p. 13). A partir de los postulados de Sechehaye (1929), el procedimiento que se sigue comienza con la coordinación, continúa con el conocimiento de las palabras principales y complementarias y concluye con la subordinación, "que ens dóna peu a [...] integrar la frase en tota la seva plenitud" (1935a, p. 14). En último lugar, tras el acceso a la frase completa, se aborda la distinción entre lo que es principal y accesorio, lo cual permite "trobar el nucli lógic esencial sense haver parlat encara de proposicions" (p. 14), además de mostrar al alumnado la importancia cognitiva y estilística que posee el orden de los elementos en la frase.

Un último aspecto de especial importancia es que tanto Galí como Caso no entienden la expresión en un sentido restringido, limitado a lo verbal, a la denotación, sino que ambos incorporan lo no verbal como parte indispensable del aprendizaje. Para Caso, 
a la frase [la] han acompañado siempre actos reveladores de esa intención que por ella misma es incapaz de traducir directamente. Si la infancia no viese hacer lo que oye decir, jamás se fijaría en una frase; y si la experiencia no le presentase juntos muchas veces el dicho y el hecho, no lograría recordar cualquiera de los dos con ocasión del otro tan instantáneamente como es preciso para poder hablar y entender al que habla $(1889$, p. 31$)$.

Por su parte, para Galí la idea fundamental de basar las enseñanzas linguiísticas en la frase viva y de entender que la lengua está hecha para la acción conlleva concebir el gesto, la expresión de la cara, las palabras dentro de la frase con sus contrastes y relaciones como formas de expresión de los procesos vivos de la persona y una enseñanza del lenguaje que no las tuviera en cuenta estaría claramente incompleta (véase Camps, 1986, pp. 48-49).

Como se ha podido apreciar, lo que estos autores pretenden es una enseñanza explícita y consciente de la gramática que sirva al alumnado para desarrollar sus capacidades verbales.

\section{Conclusiones}

En este trabajo, hemos analizado propuestas para el aprendizaje de la lengua realizadas entre la segunda mitad del siglo XIX y el primer tercio del siglo XX por Mariano Carderera, José de Caso y Alexandre Galí. Observamos que lo que se plantea, desde diferentes posturas lingüísticas y pedagógicas, es un método basado en el descubrimiento del funcionamiento de la lengua que va de lo implícito a lo explícito y en la articulación de la enseñanza de la gramática como herramienta de reflexión. Este trabajo sobre la lengua y su gramática se desarrolla principalmente a través de la conversación y el diálogo y, aunque no hemos hablado de ello, se integra en prácticas de lectura y escritura. Ello implica una reconceptualización de lo que es la gramática y una reorganización de sus contenidos, que nos aproxima, en cierta medida, a lo que en la actualidad se entiende por gramática pedagógica:

Una gramàtica per a l'ensenyament o gramàtica pedagògica ha de ser instrument d'ensenyament i d'aprenentatge de la llengua. Ha d'oferir 
al professorat els continguts i els procediments que s'adeqüen a la funció que té, és a dir, fer accessibles aquests coneixements als aprenents amb l'objectiu que desenvolupin les seves capacitats verbals, és a dir una gramàtica que ofereixi al professorat $i$ als alumnes eines per a l'activitat metalingüística (Camps y Milian, 2017, p. 221. El subrayado es nuestro).

Asimismo, un aspecto fundamental para los autores analizados es la importancia dada al uso de la lengua, desde dos perspectivas fundamentales: como dato empírico -es el análisis y la reflexión sobre el propio uso lo que conduce al conocimiento de la lengua y su gramática-, pero también como un fin; en palabras de Bréal, citado por Caso (1879, n' $\mathrm{n}^{\mathrm{o}}$ 70, p. 96), se considera que "Un niño no conoce su lengua, cuando no sabe servirse de ella para todas las necesidades de su vida". Y desde ahí, Caso indica:

[...] conocer en sí dicho lenguaje, según hemos apuntado anteriormente, y conocerlo, no de cualquier modo (que, siendo nuestro medio diario de comunicación, un conocimiento cualquiera de él todos lo tenemos), sino más y mejor de lo que es posible por solo la práctica y el uso constante; y es obvio, que, siendo toda lengua, un medio de expresión, no podremos lograr ese propósito, si, atentos a su naturaleza de medio no lo examinamos en relación con su fin (Caso, $1879,{ }^{\circ}{ }^{6} 69$, p. 215$)$.

Nos interesa destacar que Carderera, Caso y Galí no son rara avis; son excepcionales, qué duda cabe, pero están manifestando claramente el ambiente intelectual de su época. Estas ideas se encuentran en mayor o menor medida en los debates que se suceden en la prensa profesional del magisterio, en los temas que se plantean en las Conferencias pedagógicas, tanto nacionales como regionales, en las memorias de maestros...

En este trabajo, además, hemos intentado mostrar cómo los mismos temas que se discuten actualmente en Didáctica de la lengua y que preocupan a docentes e investigadores -como muestra el presente volumen- tienen importantes antecedentes históricos, pese a los cambios de paradigmas científicos. Cabe preguntarse por qué si parece que una enseñanza reflexiva basada en el uso y apoyada en la gramática 
(implícita y explícita) es beneficiosa para el aprendizaje de la lengua, parece que se sigue manteniendo un modelo de enseñanza basado en el estudio de conceptos gramaticales y el análisis morfosintáctico.

\section{Referencias bibliográficas}

Avendaño, J., y Carderera, M. (1850). Curso elemental de Pedagogía. Madrid: Establecimiento Tipográfico de A. Vicente.

Bally, Ch. (1926). Le langage et la vie. Paris: Payot.

Bargalló, M. (2007). Un viejo debate: gramática y enseñanza de la lengua. En L. Ruiz Miyares, A. Muñoz Alvarado y C. Álvarez Moreno (Eds.), Actas del X Simposio Internacional de Comunicación Social (pp. 280-284). Santiago de Cuba: Centro de Lingüística Aplicada.

Recuperado

de https://www.researchgate.net/publication/253240850_Un_viejo_debate gramatica_y_ensenanza_de_la_lengua_Conferencia_inaugural

Bastons, N., Comajoan-Colomé, Ll., Guasch, O., y Ribas, T. (2017). Les creences del professorat sobre l'ensenyament de la gramàtica a primària, secundària i en l'ensenyament d'adults. Caplletra. Revista Internacional de Filologia, 63, p. 139-164. doi: 10.7203/caplletra.63.10397

Boutan, P. (1998). De l'enseignement des langues. Michel Bréal linguiste et pédagoge. Paris: Hatier.

Calero Vaquera, M. L. (2008). Análisis lógico y análisis gramatical en la tradición española: hacia una (r)evolución de la sintaxis. Gramma-Temas, 3, 11-42.

Calero Vaquera, M. L. (2009). La recepción del análisis (lógico y gramatical) en manuales escolares del XVIII: Deudas y silencios. En J. M. García Martín, y V. Gaviño Rodríguez (Coords.), Ideas y realidades lingüísticas en los siglos XVIII y XIX (pp. 63-76). Cádiz: Universidad de Cádiz.

Calero Vaquera, M. L. (2015). Inicios y desarrollo de la gramática escolar en la tradición hispánica (siglo XIX). Ianua. Revista Philologica Romanica, 15-16, 103-119. Recuperado de http://www.romaniaminor.org/ianua/15-16_es.htm 
Camps i Mundó, A. (1986). La gramàtica a la escola básica entre els 5 i els 10 anys: algunes reflexions i propostes. Barcelona: Barcanova.

Camps i Mundó, A. (1997). Les proves de llengua en La mesura objectiva del treball escolar d'Alexandre Galí, 67 anys després de la seva publicació. En T. Ribas Seix (Coord.), L'avaluació formativa en l'àrea de llengua (pp. 209-221). Barcelona: Graó.

Camps i Mundó, A., y Milian, M. (2017). Una gramàtica per a l'ensenyament: característiques i objectius. Caplletra. Revista Internacional de Filologia, 63, 217-243. doi: 10.7203/caplletra.63.10400

Carderera y Potó, M. (1855). Diccionario de educación y métodos de enseñanza. Madrid: Imprenta de A. Vicente, vol. III.

Carderera y Potó, M. (1865). Principios de educación y métodos de enseñanza. Libro de texto para las Escuelas Normales. Madrid: Impr. del Colegio de Sordo-Mudos y de Ciegos.

Caso, J. de (1879). La enseñanza de la Lengua española. Revista de España, 68, 210-220; 69, 313-326; 70, 93-109.

Caso, J. de (1889). La enseñanza del idioma. Barcelona: Juan y Antonio Bastinos.

Comisión de Maestros de Valencia (7.4.1888). Enseñanza gramatical en las escuelas, El Magisterio Balear, 14, 1-6.

Cuesta, R. (2009). Sociogénesis de una disciplina escolar: la historia. Versión digital de la edición de Pomares-Corredor, 1997. Recuperado de http://www.nebraskaria.es/trabajos-y-publicaciones/

Fontich, X. y Camps, A. (2015). Gramática y escritura en la educación secundaria: estudio de caso sobre los conceptos de los profesores. Tejuelo, 22, 11-27. Recuperado de https://tejuelo.unex.es/issue/view/131

Fontich, X. y García Folgado, M. J. (2018). Grammar instruction in the Hispanic area: The case of Spain with attention to empirical studies on metalinguistic activity. L1. Educational Studies in Language and Literature, 18, 1-39. doi: 10.17239/L1ESLL2018.18.04.02

Galí, A. (1931a). Lliçons de llenguatge. Primer grau. Llibre del mestre. Barcelona: Editorial Pedagògica de 1'Associació Protectora de l'Ensenyança Catalana. 
Galí, A. (1931b). Lliçons de llenguatge. Primer grau. Segona Part. Barcelona: Editorial Pedagògica de l'Associació Protectora de l'Ensenyança Catalana.

Galí, A. (1935a). Introducció a la gramàtica. Instruccions per als Mestres. Barcelona: Editorial Pedagògica de 1'Associació Protectora de 1'Ensenyança Catalana.

Galí, A. (1935b). Introducció a la gramàtica. Vol. I. La frase La proposició. Barcelona: Editorial Pedagògica de l'Associació Protectora de l'Ensenyança Catalana.

García Folgado, M. J. (2012). Gramática y legislación educativa. En A. Zamorano Aguilar (ed.), Lengua y reflexión lingüística en el siglo XIX. Marcos, panorama y nuevas aportaciones (pp. 247-268). München: Lincom Europa Academic Publishers.

González Agapito, J. (1984). Alguns apunts sobre el pensament pedagògic d'Alexandre Galí. En V. Beneito (dir.), y R. M. Ríos (Coord.), L'obra pedagógica d'Alexandre Galí (pp. 33-40). Barcelona: ICE.

Jullien, M. A. (1862). Exposición del sistema de educación de Pestalozzi. Traducido por D.A.M.M.P. y anotada por Francisco Merino Ballesteros. Madrid: L. P. Villaverde.

Luzuriaga, L. (1925). El Método por Pestalozzi. Madrid: Ediciones de la Lectura

Montoro del Arco, E. T., y García Folgado, M. J. (2009). El análisis lógico y gramatical en los manuales escolares del siglo XIX (francés, castellano y latín). Quaderni dei CIRSIL, 8, 143-159.

Ministère du Commerce, de l'Industrie et des Colonies (1889). Congrès International de l'enseignement supérieur et l'enseignement secondaire. Paris: Imprimerie National.

Pestalozzi, J. H. (1801). Wie Gertrud ihre Kinder lehrt: ein Versuch den Müttern Anleitung zu geben, ihre Kinder selbst zu unterrichten, in Briefen / von Heinrich Pestalozzi. Bern/Zürich: Heinrich Geßner.

Pestalozzi, J. H. (1803). Buch der Mütter oder Anleitung für Mütter, ihre Kinder bemerken und reden zu lehren. Bern/Zürich: Heinrich Geßner / Tübingen: Cotta'schen.

Portolés, J. (1986). Medio siglo de filología española (18961952). Positivismo e idealismo. Madrid: Cátedra. 
Pozo Andrés, M. M. del (2003-2004). La Escuela Nueva en España: crónica y semblanza de un mito. Historia de la educación, 2223, 317-346.

Rabazas Romero, T. (2008). La política educativa y su repercusión en la pedagogía normalista de la segunda mitad del siglo XIX. En A. Tiana Ferrer (Ed.), El libro escolar, reflejo de intenciones políticas e influencias pedagógicas (pp. 293-305). Madrid: UNED.

Sechehaye, A. (1929). Essai sur la structure logique de la phrase. Paris: Honoré Champion.

Sornicola, R. (2014). On the history of European functionalism, La linguistique, 50(2), 7-62. Recuperado de https://www.cairnint.info/journal-la-linguistique-2014-2.htm

Sureda-García, B. (1985). Los inicios de la difusión del método de Pestalozzi en España. El papel de los diplomáticos españoles en Suiza y de la prensa periódica. Historia de la Educación, 4, 35-62.

Viñao Frago, A. (1997). La recepción de Pestalozzi en España (1900-1936). En A. Martínez Navarro, J. A. García Fraile, T. Rabazas Romero, y J. Ruiz Berrio (Coords.), La recepción de la pedagogía pestalozziana en las sociedades latinas (pp. 127-163). Madrid: Megazul-Endymion.

Vicen Ferrando, M. J. (1999). Mariano Carderera i Potó. Orígenes y desarrollo de su pensamiento pedagógico. Huesca: Instituto de Estudios Altoaragoneses.

Vilanou, C., y García Farrero, J. (2016). La pedagogía católica suiza, tres referentes: san Francisco de Sales, el P. Girard y la Universidad de Friburgo. En J. M. Hernández Díaz (Coord.), Influencias suizas en la educación española e iberoaméricana (pp. 83114). Salamanca: Universidad de Salamanca. 
Tejuelo, $n^{\circ} 35.2$ (2022), págs. 15-44. La enseñanza de la gramática como práctica reflexiva...

44 | P á g in a 